INVITED COMMENTARY

\title{
Endocrine treatment of transsexual persons: an Endocrine Society Clinical Practice Guideline: commentary from a European perspective
}

\author{
M C Meriggiola, E A Jannini ${ }^{1}$, A Lenzi $^{2}$, M Maggi $^{3}$ and C Manieri ${ }^{4}$ \\ Department of Obstetrics and Gynaecology, Centre for Reproductive Health, S. Orsola Hospital, University of Bologna, Via Massarenti 13, 40138 Bologna, \\ Italy, ${ }^{1}$ Course of Endocrinology and Medical Sexology, Department of Experimental Medicine, University of L'Aquila, L'Aquila, Italy, ${ }^{2}$ Department of \\ Medical Physiopathology, University of Rome 'La Sapienza', Rome, Italy, ${ }^{3}$ Andrology Unit, Department of Clinical Physiopathology, University of Florence, \\ Florence, Italy and ${ }^{4}$ S.C.D.U. Endocrinology and Metabolic Disease, University of Turin, Turin, Italy \\ (Correspondence should be addressed to M C Meriggiola; Email: cristina.meriggiola@unibo.it)
}

\begin{abstract}
The treatment of transsexual subjects is a challenging task for the endocrinologist who, in collaboration with the mental health professional and the surgeon, is called upon to confirm the diagnosis and adjust hormonal treatment aimed at suppressing endogenous sex hormones and to develop hormone characteristics of the desired gender. These guidelines are structured to provide evidence-based suggestions or, where evidence is lacking, expert recommendations on diagnostic procedures and hormonal treatment in adolescent and adult transsexuals, including long-term care and eligibility for surgery. The multidisciplinary approach to treatment, the additional diagnostic role of hormone administration and the need to maintain hormone levels within the physiological range are key suggestions stressed in the guidelines which are particularly important for an endocrinologist unfamiliar with this field. The need for psychological assessment before surgery is not common in many countries and should be stressed further in the guidelines. Some important issues such as time and method of hormone withdrawal before surgery together with when and which hormones should be administered after sex reassignment surgery has been completed also remain unclear.

These guidelines represent a pivotal document for endocrinologists setting a standard for the care of transsexuals and providing directions for future research.
\end{abstract}

European Journal of Endocrinology 162 831-833

Recently, the Endocrine Society published clinical practice guidelines developed by a task force of scientists with long-term experience in the field of transsexualism or gender identity disorder (1). The hormonal treatment of transsexuals is a challenging task for the endocrinologist who is called upon to administer cross-sex hormones and anti-hormones to reduce phenotypic characteristics of the biological sex and to develop those of the desired gender. Medical and surgical sex reassignments have been shown to represent effective treatments for these highly suffering people $(2,3)$. The publication of these guidelines by the pre-eminent and influential Endocrine Society testifies to the changing clinical behaviour and scientific interest towards this condition. It also represents an important step towards the elimination of prejudices and negative attitudes often present among professionals, and opens the way to rational and shared treatments.

Although general clinical suggestions from the World Professional Association for Transgender Health and other societies have been published $(4,5)$, these guidelines are specifically targeted towards endocrinologists. In the last decades, several papers have dealt with the effectiveness and safety of hormonal treatments but some aspects still need stronger evidence. In spite of the many uncertainties, especially on the long-term safety of these treatments, the authors of these guidelines struggle to offer evidence-based recommendations and suggestions based on expert opinion. These guidelines also underline areas in need of future research. The relatively small number of subjects treated in each centre represents a significant limitation to the drawing of significant conclusions on many aspects of these treatments. The development of these guidelines sets the basis for common management of these subjects, and thus will enable the creation of large databases to monitor effectiveness together with long-term risks and benefits of these hormonal regimens.

Transsexualism is a multidimensional phenomenon that requires a multidisciplinary approach in both 
the diagnostic and treatment phases (6). The multidisciplinary approach, cornerstone of the successful treatment of transsexual subjects, is well highlighted throughout the paper. Endocrinologists have to work together with mental health professionals and surgeons to provide full counselling, confirm the diagnosis, and avoid mistakes or regrets (7). Indeed, hormonal treatment not only has a therapeutic role but also is an important diagnostic tool. Subjects who live as the desired gender and experience the desired secondary sexual characteristics - the so-called real-life test - confirm appropriateness of the diagnosis and balance their decision towards the irreversible step of the surgery.

The guidelines pay special attention to the description of diagnostic procedures and treatment of adolescent transsexuals, a difficult and controversial task requiring specific expertise and great caution (8). Diagnosis difficulty of prepubertal transsexualism, the possibility of remission, and the questions regarding the safety of blocking puberty is well highlighted and discussed by the authors. Blocking puberty through GnRH analogue administration after reaching Tanner stage 2 is a wise step to confirm the diagnosis, relieve dysphoria and to avoid irreversible development of secondary sexual characteristics. If desired, this treatment is reversible on cessation of hormone administration and physiological puberty can be resumed. Otherwise, the guidelines suggest that steroid administration for development of puberty of the desired sex should be commenced on reaching 16 years of age, the recognized adult age in the majority of countries. It is essential that the young individual must be placed as much as possible in the condition in which to make a responsible decision and to fully understand the consequences of cross-sex hormonal treatment.

Hormonal treatment in adults is somehow more straightforward, even if not predictable $(9,10)$. The effects of steroid hormones, both estrogens and androgens, are primarily mediated by their receptors. An increasing body of evidence from pharmacogenomic studies now suggests that receptor polymorphism can be responsible for different responses of various subjects to steroid hormones $(11,12)$. Therefore, in a clinical setting, the role of the endocrinologist is not only to advise treatment, but also to discuss and set up common goals with the patients, and this should be stressed further. Transsexuals tend to self-administer high hormonal doses in order to achieve more rapid physical changes also in the belief of obtaining better results. It is of utmost importance that the goal of maintaining hormone levels within the physiological range of the opposite sex recommended in the guidelines is also shared with the individuals. Effects of the hormones must be clearly explained to the transsexual subject approaching treatment. It is also important that the individual is informed of which goals are impossible to achieve even with the highest hormonal dosages together with the possible risks involved. This initial 'contract' between physician and subject sets the basis for future success of the treatment.

Standard treatment of female-to-male (FtM) subjects includes testosterone administration, and the most commonly used formulations are listed in the guidelines. Male-to-female (MtF) subjects are treated with anti-androgens and oestrogens. Evidence-based recommendations suggest the use of non-synthetic oestrogens, especially in ageing people at higher risk of thromboembolic disease. It is known that steroid hormones increase the risk of venous thromboembolism (VTE), possibly through a negative effect on coagulation inhibitors. Dose, administration route and type of oestrogen compounds are highly related to thromboembolic risk. A higher incidence of VTE has been reported in transsexual people using oral ethinyl oestradiol $\left(\mathrm{EOE}_{2}\right)$ compared to those using transdermal $\mathrm{OE}_{2}$ or $17-\beta \mathrm{EE}_{2}$ (13). Therefore, $\mathrm{EOE}_{2}$ is not recommended, particularly in ageing transsexual subjects. Both steroid intake and surgery represent important risk factors for VTE, a risk that may last several weeks after major surgeries. Moreover, transsexual subjects often undergo multiple surgeries, and this may increase the risk even further. In our country (Italy), it is general practice to stop hormone intake (5) before surgery and to adopt prophylactic measures after surgery. However, there is no evidence in the literature on transsexual care, and guidelines do not provide any indication about which hormones should be suspended and how soon before and after surgery. This important issue deserves further research in an effort to provide practical indications to endocrinologists and surgeons.

Detailed monitoring plans are provided for both MtF and FtM subjects with particular attention to critical issues of adverse effects and long-term care. Guidelines indicate that these subjects carry the potential longterm risks of both sexes and must therefore undergo age recommended screening for both sexes such as prostate and mammary screening for MtF subjects.

Genital surgery is the ultimate goal of many - albeit not all - transsexuals who wish to live fully in the desired sex. Eligibility and readiness must be certified by both a mental health professional and an endocrinologist (14). In our opinion, it is very important to stress that no surgery should be performed without having this evaluation. Although in Europe this is general practice, in other parts of the world genital surgery is performed without prior psychiatric and sexological assessment and without a documented real-life experience.

The effects of hormone administration on genital organs are not completely understood. In FtM, the recommendation to undergo surgery in light of the difficulty of following these individuals with regular screening for age and sex and of the safety of the surgery seems wise, although it is not supported by any data suggesting adverse effects of testosterone administration on genital tissue $(15,16)$. 
No data are available which specifically address the issue of treatment after surgery, and no suggestions are reported in the guidelines. While no major changes are expected in the treatment of FtM subjects, the effects of continuing anti-androgen treatment after surgery in $\mathrm{MtF}$ individuals still complaining of hair growth or administering androgenic compounds in subjects with reduced sexual desire (or other androgen deprivation symptoms) remain unknown.

In conclusion, these guidelines represent an important pivotal document for endocrinologists, setting a standard for the care of transsexual people. It is hoped that they will prompt not only better and more conscious care of these patients, but will also promote additional research on the many aspects of this treatment which still remain uncertain and unclear.

\section{Declaration of interest}

The authors declare that there is no conflict of interest that could be perceived as prejudicing the impartiality of the research reported.

\section{Funding}

This research did not receive any specific grant from any funding agency in the public, commercial or not-for-profit sector.

\section{References}

1 Hembree WC, Cohen-Kettenis P, Delemarre-van de Waal HA, Gooren LJ, Meyer WJ III, Spack NP, Tangpricha V \& Montori VM. Endocrine treatment of transsexual persons: an Endocrine Society clinical practice guideline. Journal of Clinical Endocrinology and Metabolism 200994 3132-3154.

2 Cohen-Kettenis PT \& Gooren LJ. Transsexualism: a review of etiology, diagnosis and treatment. Journal of Psychosomatic Research $199946315-333$.

3 Smith YL, Van Goozen SH, Kuiper AJ \& Cohen-Kettenis PT. Sex reassignment: outcomes and predictors of treatment for adolescent and adult transsexuals. Psychological Medicine 200535 89-99.

4 Meyer WJ III, Bockting W, Cohen-Kettenis P, Coleman E, DiCeglie D, Devor H, Gooren L, Hage JJ, Kirk S, Kuiper B, Laub D, Lawrence A, Menard Y, Monstrey S, Patton J, Schaefer L, Webb A \& Wheeler CC. Harry Benjamin International Gender Dysphoria Association's The Standards of Care for Gender Identity Disorders, 6th version. 2001 Available at http://www. wpath.org/publications_standards.cfm.
5 Godano A, Maggi M, Jannini EA, Meriggiola MC, Ghigo E, Todarello O, Lenzi A \& Manieri C. SIAMS-ONIG consensus on hormonal treatment in gender identity disorder. Journal of Endocrinological Investigation 200932 857-864.

6 Fisher AD, Bandini E, Ricca V, Ferruccio N, Corona G, Meriggiola MC, Jannini EA, Manieri C, Ristori J, Forti G, Mannucci E \& Maggi M. Dimensional profile of male to female gender identity disorder: an exploratory research. Journal of Sexual Medicine, 2010 In press DOI: 10.1111/j.1743-6109. 2009.01687.x

7 Jannini EA, Lenzi A \& Wagner G. Behavioural therapy and counselling. In Andrology for the Clinician, pp 598-607. Eds WB Schill, FH Comhaire \& TB Hargreave, Berlin: Springer, 2006.

8 Cohen-Kettenis PT, Delemarre-van de Waal HA \& Gooren LJ. The treatment of adolescent transsexuals: changing insights. Journal of Sexual Medicine 20085 1892-1897.

9 Gooren LJ, Giltay EJ \& Bunck MC. Long-term treatment of transsexuals with cross-sex hormones: extensive personal experience. Journal of Clinical Endocrinology and Metabolism 2008 $9319-25$.

10 Mueller A \& Gooren L. Hormone-related tumors in transsexuals receiving treatment with cross-sex hormones. European Journal of Endocrinology 2008159 197-202.

11 Zitzmann M \& Nieschlag E. Androgen receptor gene CAG repeat length and body mass index modulate the safety of long-term intramuscular testosterone undecanoate therapy in hypogonadal men. Journal of Clinical Endocrinology and Metabolism 200792 3844-3853.

12 Nott SL, Huang Y, Fluharty BR, Sokolov AM, Huang M, Cox C \& Muyan M. Do estrogen receptor beta polymorphisms play a role in the pharmacogenetics of estrogen signaling? Current Pharmacogenomics and Personalized Medicine 20086 239-259.

13 Toorians AW, Thomassen MC, Zweegman S, Magdeleyns EJ, Tans G, Gooren LJ \& Rosing J. Venous thrombosis and changes of hemostatic variables during cross-sex hormone treatment in transsexual people. Journal of Clinical Endocrinology and Metabolism 200388 5723-5729.

14 Monstrey S, Hoebeke P, Dhont M, De Cuypere G, Rubens R, Moerman M, Hamdi M, Van Landuyt K \& Blondeel P. Surgical therapy in transsexual patients: a multi-disciplinary approach. Acta Chirurgica Belgica $2001101200-209$.

15 Perrone AM, Cerpolini S, Maria Salfi NC, Ceccarelli C, De Giorgi LB, Formelli G, Casadio P, Ghi T, Pelusi G, Pelusi C \& Meriggiola MC. Effect of long-term testosterone administration on the endometrium of female-to-male (FtM) transsexuals. Journal of Sexual Medicine 20096 3193-3200.

16 O'Hanlan KA, Dibble M \& Young-Spint M. Total laparoscopic hysterectomy for female-to-male transsexuals. Obstetrics and Gynecology $20071101096-1101$.

Received 19 January 2010

Accepted 10 February 2010 